\title{
The Role of RIP2 in p38 MAPK Activation in the Stressed Heart ${ }^{*}$
}

Received for publication, September 14, 2007, and in revised form, February 27, 2008 Published, JBC Papers in Press, February 29, 2008, DOI 10.1074/jbc.M707750200

\begin{abstract}
Sebastien Jacquet ${ }^{\ddagger}$, Yasuhiro Nishino ${ }^{\ddagger}$, Sarawut Kumphune ${ }^{\ddagger}$, Pierre Sicard ${ }^{\ddagger}$, James E. Clark ${ }^{\ddagger}$, Koichi S. Kobayashi $^{\S}$, Richard A. Flavell", Jan Eickhoff", Matt Cotten ${ }^{* *}$, and Michael S. Marber ${ }^{\ddagger 1}$

From the ${ }^{\ddagger}$ Cardiovascular Division, King's College London, The Rayne Institute, St. Thomas' Hospital,

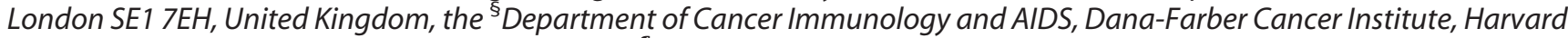
Medical School, Boston, Massachusetts 02115, the "Department of Immunobiology, Howard Hughes Medical Institute, Yale University School of Medicine, New Haven, Connecticut 06520, "GBC Biotech, München D82152, Germany, and **Medical Research Council Laboratories, P. O. Box 273, Banjul, Gambia
\end{abstract}

The activation of p38 MAPK by dual phosphorylation aggravates myocardial ischemic injury and depresses cardiac contractile function. SB203580, an ATP-competitive inhibitor of p38 MAPK and other kinases, prevents this dual phosphorylation during ischemia. Studies in non-cardiac tissue have shown receptor-interacting protein 2 (RIP2) lies upstream of p38 MAPK, is SB203580-sensitive and ischemia-responsive, and aggravates ischemic injury. We therefore examined the RIP2p38 MAPK signaling axis in the heart. Adenovirus-driven expression of wild-type RIP2 in adult rat ventricular myocytes caused robust, SB203580-sensitive dual phosphorylation of p38 MAPK associated with activation of p38 MAPK kinases MKK3, MKK4, and MKK6. The effect of SB203580 was recapitulated by unrelated inhibitors of RIP2 or the downstream MAPK kinase kinase, TAK1. However, overexpression of wild-type, kinasedead, caspase recruitment domain-deleted, or kinase-dead and caspase recruitment domain-deleted forms of RIP2 had no effect on the activating dual phosphorylation of p38 MAPK during simulated ischemia. Similarly, p38 MAPK activation and myocardial infarction size in response to true ischemia did not differ between hearts from wild-type and RIP2 null mice. However, both p38 MAPK activation and the contractile depression caused by the endotoxin component muramyl dipeptide were attenuated by SB203580 and in RIP2 null hearts. Although RIP2 can cause myocardial p38 MAPK dual phosphorylation in the heart under some circumstances, it is not responsible for the SB203580-sensitive pattern of activation during ischemia.

There is overwhelming evidence that the activation of p38 MAPK $^{2}$ during prolonged myocardial ischemia accelerates injury (see Refs. 1 and 2 for review). Thus, in theory at least, inhibitors of p38 MAPK have therapeutic potential in ischemic

\footnotetext{
* This work was supported by Wellcome Trust Grant 074653 and British Heart Foundation Grant 07/073/23432 (to M. S. M.). The costs of publication of this article were defrayed in part by the payment of page charges. This article must therefore be hereby marked "advertisement" in accordance with 18 U.S.C. Section 1734 solely to indicate this fact.

${ }^{1}$ To whom correspondence should be addressed. E-mail: mike.marber@ kcl.ac.uk.

${ }^{2}$ The abbreviations used are: MAPK, mitogen-activated protein kinase; CARD, caspase recruitment domain; MDP, muramyl dipeptide; MKK, mitogen-activated protein kinase kinase; RIP2, receptor-interacting protein 2; TAK1, transforming growth factor- $\beta$-activated protein kinase 1 ; ARVM, adult rat ventricular myocyte; HA, hemagglutinin; KHB, Krebs-Henseleit buffer; WT, wild-type.
}

heart disease. However, enthusiasm for "blanket" pharmacological inhibition of p38 MAPK is tempered by the facts that it is involved in innumerable biological processes (3), its major isoform is essential for early embryogenesis (4), and hepatic toxicity has curtailed the development of at least three clinical trial programs (5). These drawbacks have increased interest in the mechanisms controlling p38 activation in the hope they will reveal new, less crucial, therapeutic targets (5).

The activity of p38 MAPK is controlled by dual phosphorylation of the $\mathrm{Thr}^{180}-\mathrm{Gly}^{181}-\mathrm{Tyr}^{182}$ motif within the activation loop/lip (3). The traditional view is that this dual phosphorylation event is achieved by upstream, dual specificity MAPK kinases or MKKs (3, 5). SB203580 (4-(4-fluorophenyl)-2-(4methylsulfinylphenyl)-5-(4-pyridyl)- $1 H$-imidazole), a pyridinyl imidazole, is the most widely used pharmacological inhibitor of p38 MAPK. Because it occupies the catalytic site without inhibiting upstream MKKs, it should inhibit the phosphorylation events downstream of p38 MAPK without inhibiting the dual phosphorylation of p38 MAPK itself. Nonetheless, there are numerous examples where SB203580 and other pyridinyl imidazoles diminish the dual phosphorylation of p38 MAPK during ischemia and related myocardial stresses (see Fig. 2 of Ref. 6, Fig. 4 of Ref. 7, Fig. 2 of Ref. 8, Fig. 3 of Ref. 9, Fig. 10 of Ref. 10, Fig. 4 of Ref. 11, Fig. 3 of Ref. 12, Fig. 5 of Ref. 13, and Fig. $5 A$ of Ref. 14). Thus, unlike most stresses (15), myocardial ischemia seems to reproducibly cause an SB203580-sensitive form of p38 MAPK dual phosphorylation that must indicate a mechanism of activation that occurs under this circumstance. One explanation for these robust observations is that SB203580 inhibits a kinase upstream of p38 MAPK involved in its activation by transphosphorylation during ischemia. Once identified, inhibition of such a kinase may prevent p38 MAPK activation during ischemia but not under other circumstances possibly required for homeostasis.

Godl et al. (16) performed an extensive affinity screen in which they identified other kinases binding to pyridinyl imidazoles, among which was RIP2, also known as RICK (Rip-like interacting caspase-like apoptosis regulatory protein kinase) or CARDIAK (CARD-containing interleukin-1 $\beta$-converting enzyme-associated kinase) $(16,17)$. RIP2 is a likely candidate to mediate the SB203580-sensitive dual phosphorylation of p38 during ischemia because it is highly expressed in the heart (18), is more sensitive to SB203580 than p38 $\alpha$ MAPK (16), has been implicated in ischemia-initiated cell death in neurons (19), and lies upstream of p38 MAPK (20). The principal purpose of this 
study was to explore whether RIP2 is responsible for the SB203580-sensitive activating dual phosphorylation of p38 MAPK and injury during myocardial ischemia.

\section{MATERIALS AND METHODS}

Products-Antibodies recognizing the dual phospho-Thr ${ }^{180}$ / $\mathrm{Tyr}^{182}$ form of $\mathrm{p} 38$ MAPK (catalog no. 9211), total p38 MAPK (catalog no. 9212), and HA tag (catalog no. 2367) were from Cell Signaling Technology. Antibodies recognizing RIP2 (catalog no. ALX-804-139) and the phospho-Ser ${ }^{189}$ forms of MKK3 and MKK6 (catalog no. 7994) were from Alexis and Santa Cruz Biotechnology, respectively. SB203580 (catalog no. S8307) was obtained from Sigma, myelin basic protein (catalog no. 13-110) was from Upstate, PP2 (catalog no. 529576) was from Merck, TAK1 inhibitor (5Z-7-oxozeaenol; catalog no. NP-009245) was from Analyticon Discovery, and muramyl dipeptide (MDP) (catalog no. TLR-MDP) was from Invitrogen.

Perfusion of Isolated Murine Hearts-All experiments were performed in accordance with the United Kingdom Home Office "Guidance on the Operation of Animals (Scientific Procedures) Act 1986."

Male RIP2 null (21) and wild-type mice were anesthetized with intraperitoneal pentobarbital $(300 \mathrm{mg} / \mathrm{kg})$ and heparin (150 units). Hearts were rapidly isolated and placed in ice-cold modified Krebs-Henseleit buffer (KHB; $118.5 \mathrm{mmol}$ of $\mathrm{NaCl}$, $25.0 \mathrm{mmol}$ of $\mathrm{NaHCO}_{3}, 4.75 \mathrm{mmol}$ of $\mathrm{KCl}, 1.18 \mathrm{mmol}$ of $\mathrm{KH}_{2} \mathrm{PO}_{4}, 1.19 \mathrm{mmol}$ of $\mathrm{MgSO}_{4}, 11.0 \mathrm{mmol}$ of D-glucose, and 1.4 $\mathrm{mmol}$ of $\left.\mathrm{CaCl}_{2}\right)$. The excised heart was mounted onto a Langendorff apparatus and retrogradely perfused at a constant pressure of $80 \mathrm{~mm} \mathrm{Hg}$ with $\mathrm{KHB}$ equilibrated with $95 \% \mathrm{O}_{2}$ and $5 \%$ $\mathrm{CO}_{2}$ at $37^{\circ} \mathrm{C}$. A fluid-filled balloon inserted into the left ventricle monitored contractile function. The balloon was gradually inflated until the end diastolic pressure was between 2 and 8 $\mathrm{mm} \mathrm{Hg}$. Atrial pacing was performed at 580 beats $/ \mathrm{min}$ with a 0.075-mm silver wire (Advent). Coronary flow was measured by timed collection of perfusate. More detailed methods and exclusion and inclusion criteria ware as described previously $(22,23)$.

Infarction Assessment in Isolated Murine Hearts-Following a 30 -min stabilization, all hearts underwent $30 \mathrm{~min}$ of global ischemia followed by $2 \mathrm{~h}$ of reperfusion. Electrical pacing was stopped when contraction ceased during ischemia and was restarted $30 \mathrm{~min}$ into reperfusion. After $2 \mathrm{~h}$ of reperfusion, hearts were perfused for $1 \mathrm{~min}$ with $5 \mathrm{ml}$ of $1 \%$ triphenyltetrazolium chloride in phosphate-buffered saline and then placed in an identical solution at $37^{\circ} \mathrm{C}$ for $10 \mathrm{~min}$. The atria were removed, and the hearts were blotted dry, weighed, and stored at $-20^{\circ} \mathrm{C}$ for up to 1 week. Hearts were then thawed, placed in $2.5 \%$ glutaraldehyde for $1 \mathrm{~min}$, and set in $5 \%$ agarose. The agarose heart blocks were sectioned from apex to base in $0.75-\mathrm{mm}$ slices using a Vibratome (Agar Scientific). After sectioning, slices were placed overnight in $10 \%$ formaldehyde at room temperature before transferring to phosphate-buffered saline for an additional day at $4{ }^{\circ} \mathrm{C}$. Sections were then compressed between glass plates $(0.75 \mathrm{~mm}$ apart) and scanned (Epson Model G850A). After magnification, planimetry was carried out using image analysis software (Adobe Photoshop 7.0), and the surface areas of the whole and triphenyltetrazolium chlo- ride-negative myocardium were transformed to volume by multiplication with tissue thickness. Within each heart, after the summation of individual slices, triphenyltetrazolium chloride-negative infarction volume was expressed as a percentage of heart volume. All analyses of infarct size were done by an investigator who was blinded with regard to the group assignments.

Adult Rat Ventricular Myocyte (ARVM) Culture-ARVMs were isolated as described previously (23) and washed with M199 medium with added penicillin (100 IU/ml) and streptomycin $(100 \mathrm{IU} / \mathrm{ml})$. The cell suspension was centrifuged at $100 \times g$ for $2 \mathrm{~min}$ to pellet the myocytes, which were then resuspended in complete M199 medium (M199 medium with added penicillin $(100 \mathrm{IU} / \mathrm{ml})$, streptomycin $(100 \mathrm{IU} / \mathrm{ml})$, L-carnitine $(2 \mathrm{mM})$, creatine $(5 \mathrm{mM})$, and taurine $(5 \mathrm{~mm}))$ and placed in laminin-coated 6-well plates prior to incubation in $5 \% \mathrm{CO}_{2} /$ room air at $37^{\circ} \mathrm{C}$. After $1 \mathrm{~h}$, the medium was aspirated, leaving only adherent cells, and fresh prewarmed complete M199 medium was added with specified adenoviral vectors.

Adenoviral Transfection of ARVMs-Adenoviruses encoding HA-tagged forms of wild-type RIP2 (WT-RIP2), kinase-dead RIP2 (DN-RIP2; K47R), CARD-deleted RIP2 (DC-CARD; residues 1-353), and kinase-dead + CARD-deleted RIP2 (DN/DCRIP2) were as described previously (24) and were added at a multiplicity of infection of 50 when not otherwise specified (providing $\sim 95 \%$ efficiency of transfection). Cells were exposed to virus for $1 \mathrm{~h}$ prior to washing and incubated in virus-free complete M199 medium for $24 \mathrm{~h}$.

Simulated Ischemia Protocol-Simulated ischemia was induced by exposing ARVMs for the specified duration to modified KHB (137 mM NaCl, $3.8 \mathrm{~mm} \mathrm{KCl}, 0.49 \mathrm{~mm} \mathrm{MgCl}_{2}, 0.9 \mathrm{~mm}$ $\mathrm{CaCl}_{2}$, and $4.0 \mathrm{~mm}$ HEPES) with $10 \mathrm{~mm}$ 2-deoxyglucose, $20 \mathrm{~mm}$ sodium lactate, and $1 \mathrm{~mm}$ sodium dithionite at $\mathrm{pH} 6.5$ (25). When specified, cells were exposed to $0.1 \mu \mathrm{M}$ SB203580 for 60 min prior to and during simulated ischemia. Following simulated ischemia, the buffer was removed, and ARVMs were quickly frozen and stored in liquid nitrogen.

RIP2 Immunoprecipitation and Kinase Assay-ARVMs were infected with HA-WT-RIP2 adenovirus; exposed $24 \mathrm{~h}$ later, when indicated, to SB203580 at $0.1 \mu \mathrm{M}$ for $1 \mathrm{~h}$ prior to washing with ice-cold phosphate-buffered saline; and quickly frozen in liquid nitrogen. The cells were lysed in buffer containing $0.5 \%$ Nonidet P-40, and after centrifugation to clear the sample, the supernatant was collected, and anti-HA antibody was added prior to overnight agitation at $4{ }^{\circ} \mathrm{C}$. The RIP2-antibody complex was pulled down using protein $\mathrm{G}$ beads coupled to agarose. After extensive washes, the pellet was resuspended in kinase buffer with the myelin basic protein as a substrate, $\left[{ }^{32} \mathrm{P}\right] \mathrm{ATP}(5$ $\mu \mathrm{Ci} /$ assay), and $0.1 \mu \mathrm{M}$ SB203580 when specified, and the mixture was incubated at $30^{\circ} \mathrm{C}$ for $30 \mathrm{~min}$. The reaction was stopped by the addition of boiling SDS-PAGE sample buffer. The incorporation of ${ }^{32} \mathrm{P}$ in myelin basic protein was measured by autoradiography after separation on a $12 \%$ acrylamide gel.

Polyacrylamide Gel Electrophoresis and Western Blot Analysis-ARVMs and hearts were homogenized and solubilized in SDS-PAGE sample buffer as described previously (22, 23). Proteins were separated on 10 or $12 \%$ SDS-polyacrylamide gels; transferred to polyvinylidene difluoride membranes, 

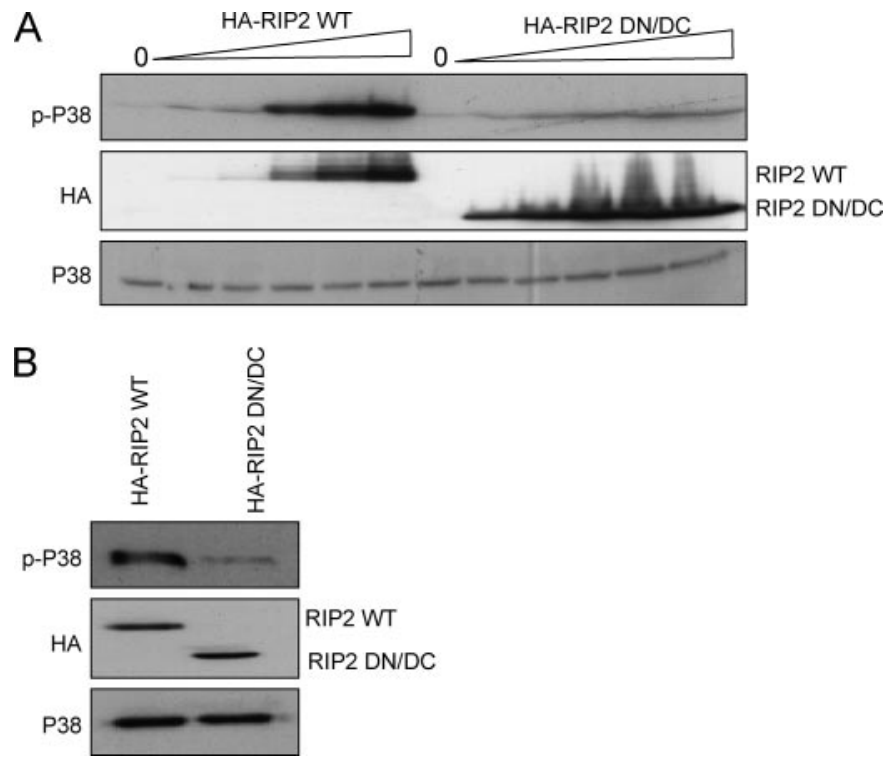

FIGURE 1. p38 MAPK is activated by RIP2 in cardiomyocytes. $A$, ARVMs were exposed to adenoviral vectors encoding full-length HA-tagged RIP2 (WT-RIP2) or kinase-dead and CARD-deleted tagged RIP2 (DN/DC-RIP2) at increasing multiplicities of infection $(0-100) .24 \mathrm{~h}$ after exposure, cells were harvested, and the activation of p38 MAPK ( $p$-P38) was assessed by Western blotting. $B$, the format was as in $A$, but proteins were from an embryonic rat heart-derived cell line ( $\mathrm{H} 9 \mathrm{c} 2)$ using a multiplicity of infection of 50.

which were blocked for $2 \mathrm{~h}$ with $5 \%$ nonfat milk plus $1 \%$ bovine serum albumin in Tris-buffered saline ( $\mathrm{pH} 7.4$ ) containing $0.1 \%$ Triton; and probed overnight at $4{ }^{\circ} \mathrm{C}$ with the appropriate primary antibody. After washing and exposure for $2 \mathrm{~h}$ at room temperature to horseradish peroxidase-conjugated secondary antibody, antibody-antigen complexes were visualized by enhanced chemiluminescence.

Statistical Analysis-Results are expressed as mean \pm S.D. Data sets were analyzed by one-way (Figs. $5 B, 6 C$, and $7 B$ ) or two-way (Figs. $5 A ; 6, A$ and $B$; and $7 A$ ) analysis of variance followed by Tukey's multiple comparison test. A value of $p<$ 0.05 was considered statistically significant.

\section{RESULTS}

Adenoviral Expression of RIP2 Increases the Dual Phosphorylation of 38 MAPK-To determine whether RIP2 lies upstream of p38 MAPK in cardiac myocytes, ARVMs were exposed to increasing concentrations of adenovirus encoding full-length RIP2 (WT-RIP2) or CARD-deleted and kinase-dead RIP2 (DN/ DC-RIP2) (24). WT-RIP2 was able to induce a robust activation of p38 MAPK (Fig. 1A), but a similar level of ectopic expression of DN/DC-RIP2 caused no activation. Similar results were obtained in other cells, including embryonic rat heart-derived H9c2 (Fig. $1 B$ ) and adult rat ventricular fibroblasts (data not shown).

RIP2 Kinase Activity and p38 MAPK Activation by RIP2 Are Sensitive to Pharmacological Inhibition by SB203580 and PP2-Godl et al. (16) reported that RIP2 is exquisitely sensitive to inhibition by SB203580. To document the sensitivity of RIP2 to inhibition by SB203580, we performed a kinase assay on WT-RIP2 expressed in ARVMs, followed by immunoprecipitation using the HA tag. The presence of $0.1 \mu \mathrm{M}$ SB203580 in the kinase buffer largely prevented the incorpora-
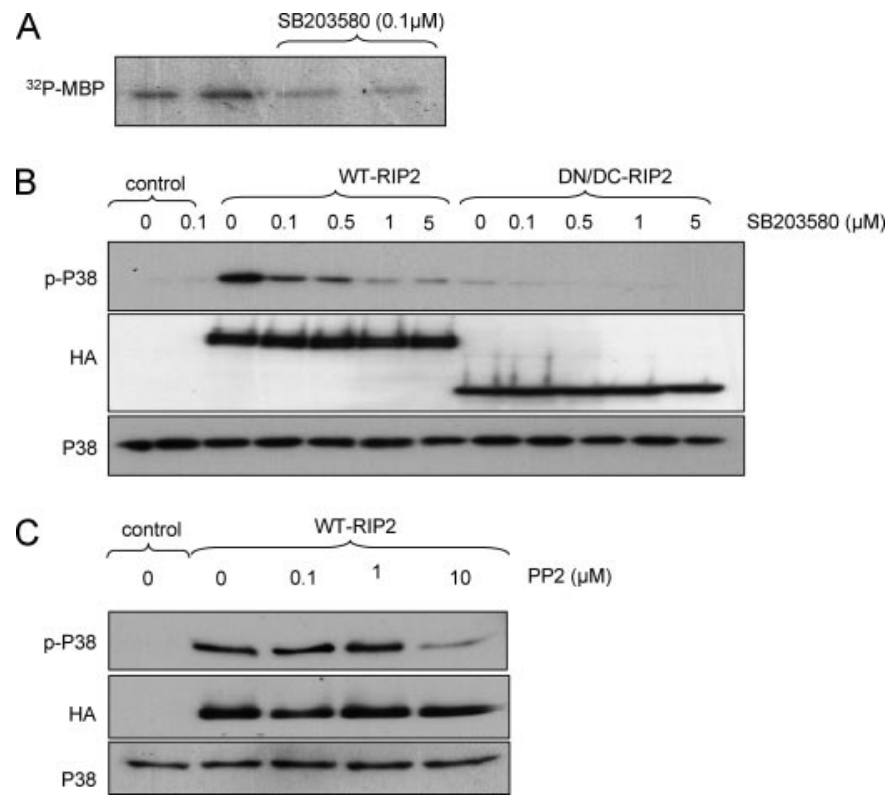

FIGURE 2. Pharmacological inhibition of RIP2 by SB203580 and PP2. $A$, an in vitro kinase assay of WT-RIP2 immunoprecipitated from ARVMs $24 \mathrm{~h}$ after exposure to the encoding adenovirus at a multiplicity of infection of 50 is shown. Kinase activity was assayed by incorporation of $\left[\gamma^{-32}\right.$ P]ATP into myelin basic protein (MBP) in the presence and absence of SB203580. B, ARVMs were transfected with adenoviral vectors encoding WT-RIP2 or DN/DC-RIP2, and after $24 \mathrm{~h}$, they were exposed to the indicated concentrations of SB203580 for $1 \mathrm{~h}$ before proteins were harvested and processed as described in Fig. $1 A$. p-P38, p38 MAPK. C, ARVMs were prepared as described in $B$ but exposed to increasing concentrations of PP2 (0-10 $\mu \mathrm{M})$ instead of SB203580.

tion of ${ }^{32} \mathrm{P}$ into myelin basic protein (Fig. $2 A$ ). To determine whether the inhibition of RIP2 kinase activity by SB203580 diminishes endogenous p38 MAPK dual phosphorylation, we performed a dose-response study on ARVMs expressing WTRIP2 or DN/DC-RIP2 (Fig. 2B). As expected, SB203580 prevented RIP2-induced p38 MAPK activation. It is important to note that SB203580 inhibited p38 MAPK phosphorylation at a concentration 2 orders of magnitude lower than that commonly used to inhibit p38 MAPK catalytic activity in intact cells $(10 \mu \mathrm{M})$. At this concentration, it is unlikely that our observation is the result of the inhibition of another mechanism of SB203580-sensitive p38 dual phosphorylation, viz. p38 autophosphorylation (26). However, to rule out this possibility, we exposed transfected ARVMs to PP2, which inhibits RIP2 and Src kinase activity (20), and similarly prevented p38 activation by RIP2 (Fig. 2C). In conclusion, these results suggest that RIP2 lies upstream of p38 MAPK in an SB203580- and PP2-sensitive manner.

Characterization of the Domains of RIP2 Responsible for $p 38$ MAPK Activation-RIP2 was originally described as a serine/ threonine kinase implicated in NF- $\kappa \mathrm{B}$ activation and apoptosis (27). RIP2 is composed of an N-terminal kinase domain followed by an intermediate region and a C-terminal CARD. The physiological role of the RIP2 kinase domain in isolation is uncertain because autophosphorylation of Ser ${ }^{176}$ on RIP2 (28) is the only substrate yet identified, and RIP2 has been proposed to function primarily as a scaffolding protein (27). We therefore attempted to characterize the individual roles of the CARD and kinase domain in the activation of p38 MAPK in ARVMs by using our previously described adenoviral constructs lacking 
A

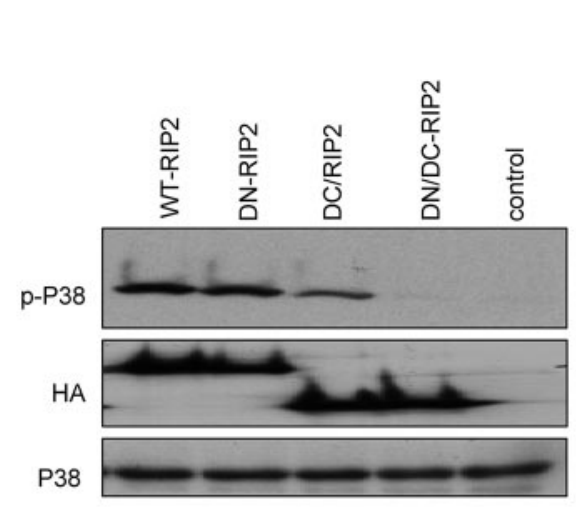

B

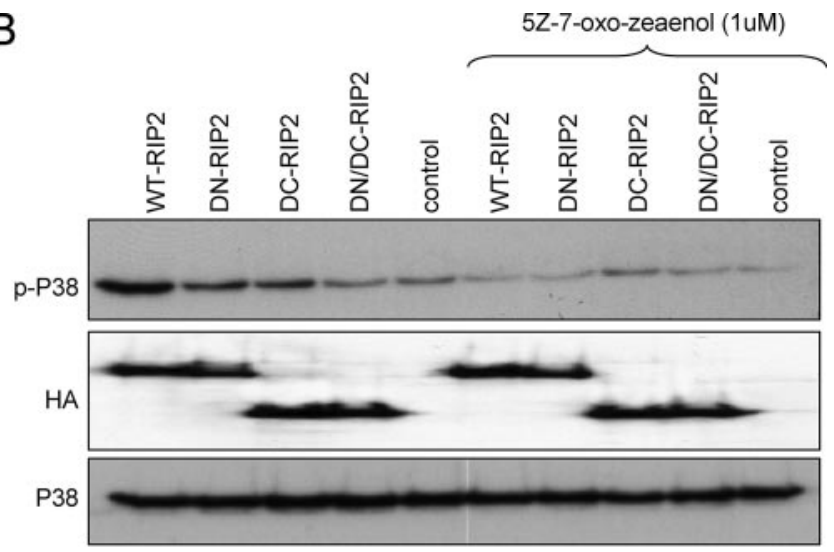

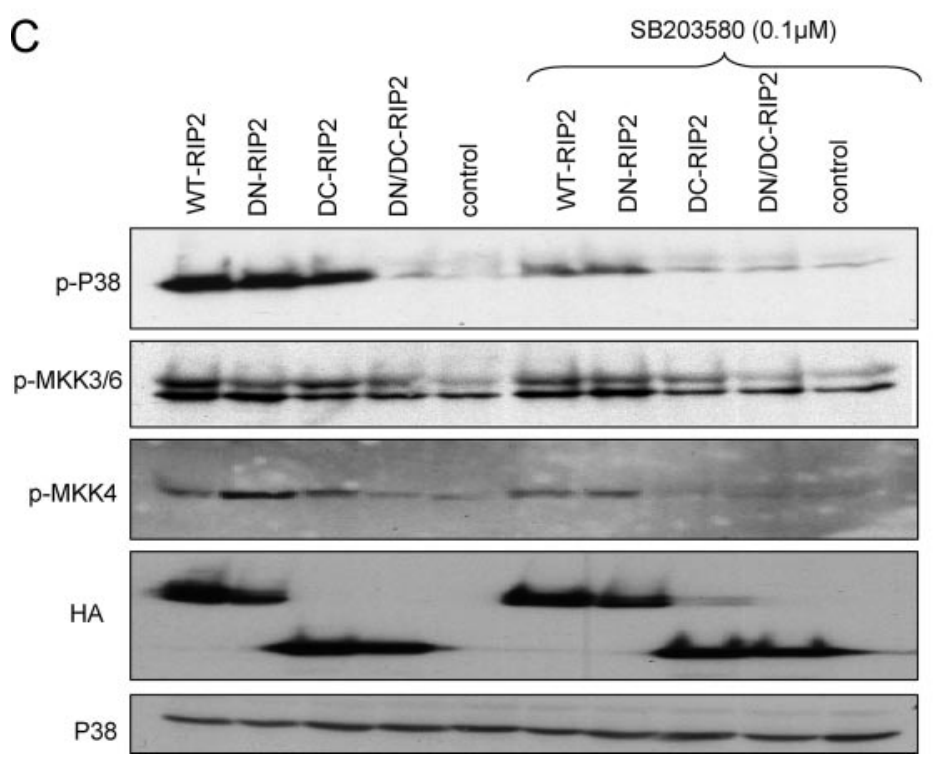

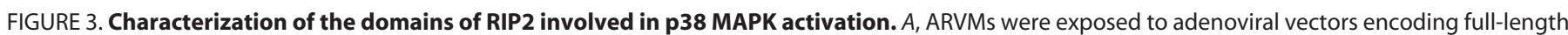

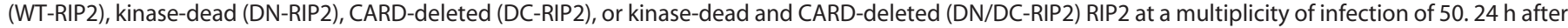

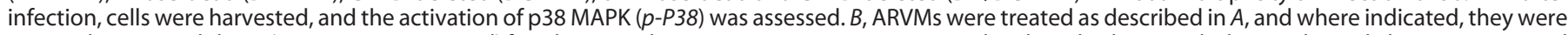

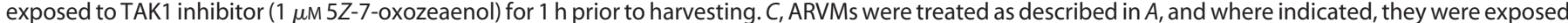

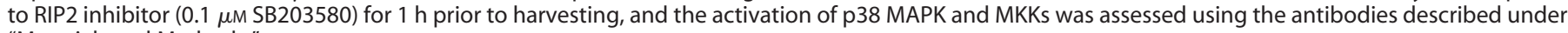
"Materials and Methods."

kinase activity (DN-RIP2) or CARD (DC-RIP2) as well as kinase activity together with CARD (DN/DC-RIP2) (Fig. 3A) (24). Expression of the kinase-dead RIP2 form resulted in robust $\mathrm{p} 38$ MAPK dual phosphorylation that appears similar to that seen with WT-RIP2, suggesting that kinase activity is not the major contributor. This was confirmed by deletion of CARD alone (DC-RIP2), which diminished p38 MAPK phosphorylation. Surprisingly, this was further diminished in DN/DC-RIP2-expressing cells, suggesting that in the absence of CARD, kinase activity contributes to p38 MAPK dual phosphorylation, but in the presence of CARD, it has a minor role.

To better understand the mechanisms responsible for this pattern of p38 MAPK dual phosphorylation, we examined the activating phosphorylations within the archetypal p38 MAPK upstream kinases TAK1 (29) and MKK (3). To evaluate whether TAK1 plays a role in the activation of p38 MAPK by RIP2, we treated cells with a TAK1 inhibitor, 5Z-7-oxozeaenol (Fig. 3B) (29). In the presence of the TAK1 inhibitor, there was markedly reduced activation of p38 MAPK by RIP2, indicating that RIP2 is acting through TAK1, confirming similar results previously obtained in HEK293 cells $(20,27,29)$. Then we further characterized the signaling pathway leading to the activation of p38 MAPK by RIP2 by studying the possible role of the MKK proteins (Fig. 3C). When cells express WT-RIP2, there is robust activation of MKK 6 and of MKK3 to a lesser extent. Interestingly, by comparing the individual mutants, it seems likely that RIP2 kinase activity is responsible for the activation of MKK6 and MKK3 (compare lanes 1 and 2 in Fig. 3C) and that CARD is responsible for the activation of MKK4 (compare lanes 2 and 4). We confirmed the role of the MKKs in the RIP2 pathway by exposing the cells to the RIP2 inhibitor SB203580 at $0.1 \mu \mathrm{M}$, and as expected, we observed decreased MKK6 and MKK4 activation (Fig. 3C). However, surprisingly, the effect of pharmacological inhibition with SB203580 on p38 MAPK dual phosphorylation was greater than the effect of the absence of kinase activity. Moreover, SB203580 haf a marked added effect on p38 MAPK dual phosphorylation caused by exposure to kinase-dead RIP2, confirming an action independent of kinase inhibition. The mechanisms that underlie this observation are worthy of fur- 
ther exploration and include SB203580 inhibiting endogenous RIP2, binding to RIP2, and interfering with downstream kinase-independent signaling and/or inhibiting other RIP2-dependent kinase signals.

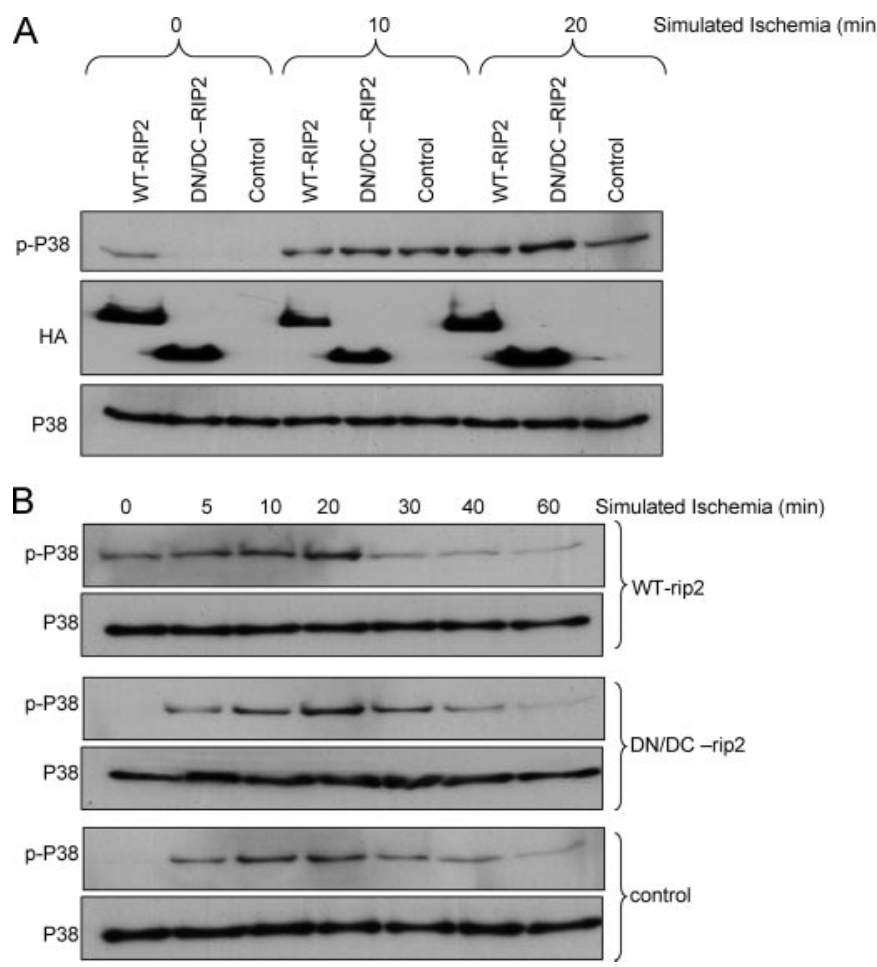

FIGURE 4. Role of RIP2 in p38 MAPK activation during simulated ischemia of ARVMs. $A$ and $B, A R V M s$ were exposed to adenoviruses encoding WT-RIP2 or DN/DC-RIP2 for $24 \mathrm{~h}$ before being subjected to simulated ischemia for the indicated duration. Cells were then harvested, and p38 MAPK ( $p-P 38)$ activation was assessed.
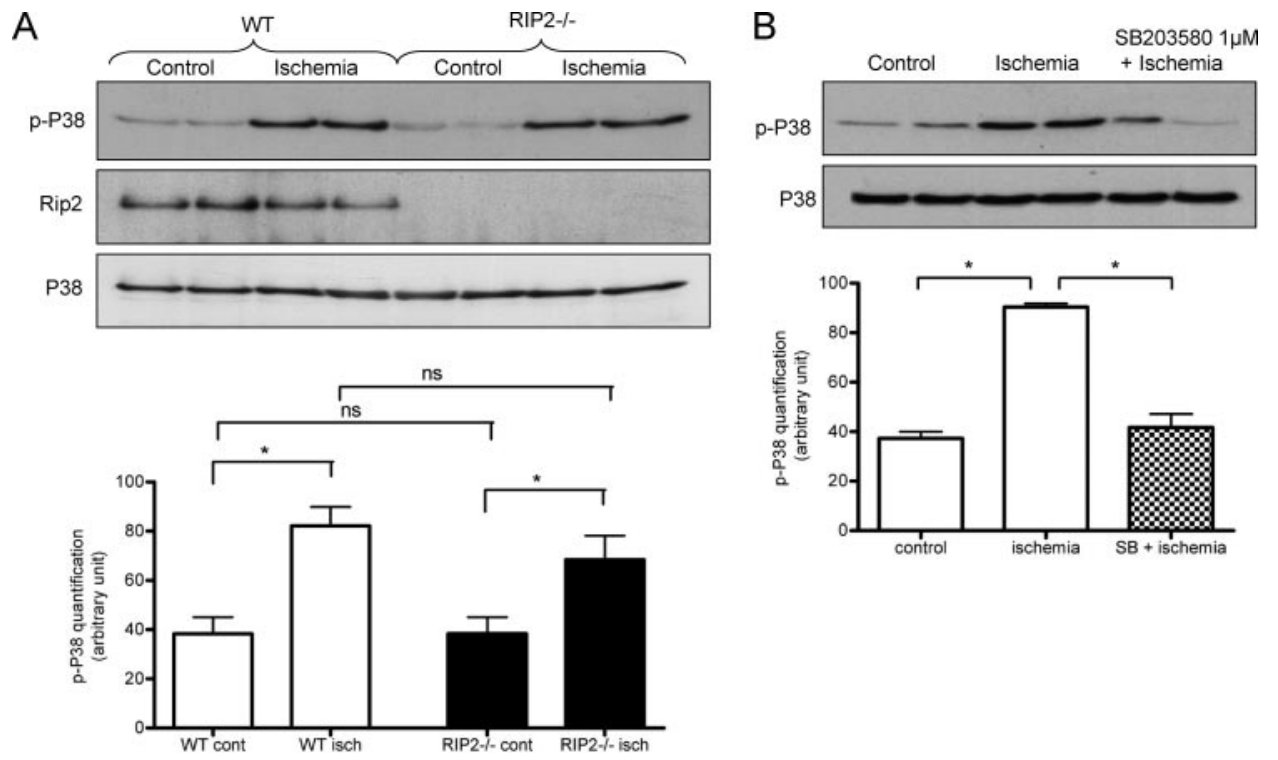

FIGURE 5. Role of RIP2 in the activation of p38 MAPK during global ischemia of the isolated mouse heart. $A$, hearts from wild-type $(n=5)$ and RIP2-deficient (RIP2 $\left.{ }^{-1-} ; n=5\right)$ mice were subjected to 30 min of aerobic perfusion and $10 \mathrm{~min}$ of global ischemia (isch) or $40 \mathrm{~min}$ of aerobic perfusion (control (cont)) prior to snapfreezing and harvesting proteins. The upper panel is a representative immunoblot of 8 hearts, and the lower panel is a quantification of band density from all 20 hearts. $n s$, not significant; $p$-P38, p38 MAPK. $B$, hearts from $\mathrm{RIP2}^{-\prime-}$ mice ( $n=5$ /group) were perfused as described in A. As indicated, one group was exposed to SB203580 $(S B)$ in the perfusate for the 10 min immediately preceding the 10-min period of global ischemia. The upper panel is a representative immunoblot of 6 hearts, and the lower panel is a quantification of band density from all 15 hearts. ${ }^{*}, p<0.05$ ( $n=5$ in each group).
In summary, RIP2 overexpression in ARVMs results in robust and SB203580-sensitive dual phosphorylation of p38 MAPK. This pattern is identical to that seen during myocardial ischemia $(6-14)$ and is consistent with our hypothesis that it is the kinase responsible for such activation and consequent injury during myocardial infarction.

RIP2 Does Not Cause p38 MAPK Activation in ARVMs Subjected to Simulated Ischemia-ARVMs expressing WT-RIP2 and DN/DC-RIP2 were subjected to simulated ischemia of a sufficient duration to activate p38 MAPK (Fig. 4A). As expected, 10 or 20 min of simulated ischemia caused robust RIP2. To be certain that RIP2 does not cause a temporal shift in the pattern of p38 MAPK dual phosphorylation in response to simulated ischemia, we examined earlier and later time points (Fig. 4B), but there remained no discernible effect. However, simulated ischemia differs substantially from true ischemia (30), and given the encouraging findings linking RIP2 to an SB203580-sensitive form of $\mathrm{p} 38$ activation, we therefore further examined our hypothesis in the whole heart subjected to true ischemia. Curine Heart-The retrogradely perfused mouse inderlying the SB203580 sensitivity of p38 MA phorylation during ischemia $(22,31,32)$. We there from a RIP2-deficient line previously developed and described by Kobayashi et al. (21).

Wild-type and RIP2 null hearts did not differ at base line in terms of weight or contractile parameters. After $10 \mathrm{~min}$ of global ischemia (Fig. 5A), p38 MAPK became strongly dual-phosphorylated in the isolated mouse heart (22). However, neither basal nor ischemic p38 MAPK dual phosphorylation was discernibly different between hearts harvested from wild-type and RIP2 null mice. To confirm that the SB203580-sensitive dual phosphorylation of p38 MAPK seen during myocardial ischemia is definitely independent of RIP2, we exposed RIP2 null hearts to intra-ischemic SB203580 (Fig. $5 B)$. Despite the absence of RIP2, SB203580 still attenuated p38 MAPK dual phosphorylation during ischemia.

Finally, to ensure that the findings in the RIP2 null mouse hearts were not the result of altered sensitivity to ischemia, we assessed response to $30 \mathrm{~min}$ of global ischemia (Fig. 6). Neither histochemi- 
A

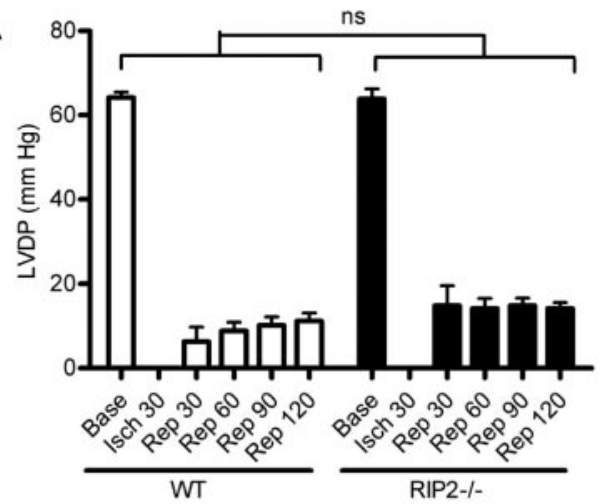

C

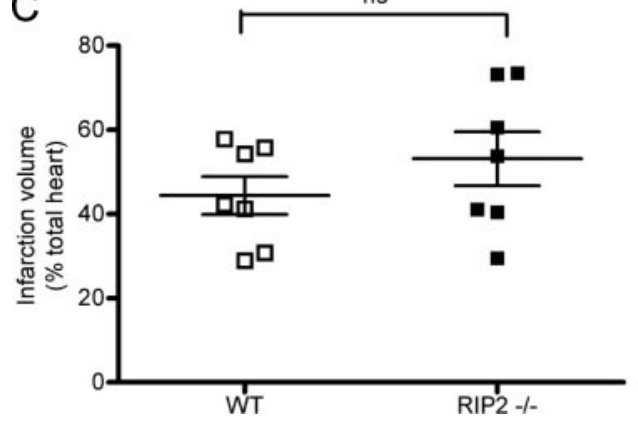

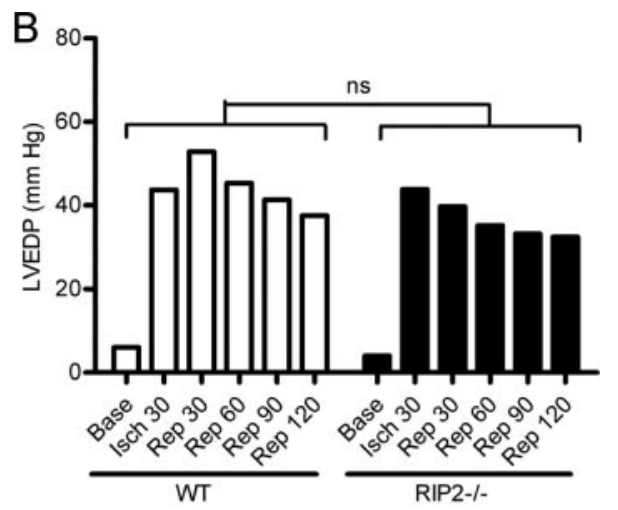

was associated with an abolition of the activating dual phosphorylation of p38 MAPK. In conclusion, both genetic and pharmacological interventions suggest that RIP2 is crucial to MDP-induced contractile dysfunction and the activating dual phosphorylation of p38 MAPK.

\section{DISCUSSION}

We have demonstrated that RIP2 can activate p38 MAPK in adult rat ventricular myocytes, signaling via TAK1 and MKK3, MKK4, and MKK6, and that this pattern of activation is sensitive to two structurally unrelated RIP2 kinase inhibitors, SB203580 and PP2. However, overexpression of wildtype RIP2 does not enhance p38 MAPK activation in adult rat ventricular myocytes subjected to simulated ischemia. Similarly, under this circumstance, overexpression of kinase-dead and CARDdeleted RIP2 does not diminish activation. Finally, the activating dual phosphorylation of p38 MAPK during myocardial ischemia occurs unhindered in RIP2 null hearts and remains sensitive to SB203580.

cally assessed infarct size (Fig. $6 C$ ) nor post-ischemic recovery of left ventricular contractile performance (Fig. 6, $A$ and $B$ ) was altered by the absence of RIP2.

RIP2 Does Cause 38 MAPK Activation and Contractile Depression in Murine Hearts Exposed to Muramyl DipeptideMDP is a component of bacterial cell walls that is thought to signal through a RIP2/p38-dependent nucleotide-binding oligomerization domain receptor pathway in non-cardiac tissue (20). The nucleotide-binding oligomerization domain is present in the heart (33), and MDP contributes to septic shock (34), which is in part the result of depressed cardiac contractility. The activation of myocardial p38 MAPK is known to depress cardiac contractility $(23,35)$. We therefore reasoned that although RIP2 was not involved in ischemia-induced contractile dysfunction and infarction, it may contribute to contractile dysfunction induced by MDP. After $20 \mathrm{~min}$, wild-type hearts exposed to KHB containing MDP $(50 \mu \mathrm{g} / \mathrm{ml})$ exhibited a $33 \%$ reduction in developed pressure (Fig. $7 A$ ). This was significantly greater than the contractile change in time-matched control hearts or similarly exposed RIP2 null hearts $(p<0.001)$. Furthermore, the preserved contractile function of RIP2 null hearts was accompanied by reduced p38 MAPK dual phosphorylation (Fig. 7B). To confirm the role of RIP2 in an SB203580sensitive form of $\mathrm{p} 38$ MAPK dual phosphorylation, we exposed hearts to SB203580 $10 \mathrm{~min}$ prior to and during the MDP perfusion period (Fig. 7C). As expected, SB203580 prevented the contractile dysfunction induced by MDP. This beneficial effect
Thus, although RIP2 overexpression recapitulates the pattern of 338 MAPK activation seen with myocardial ischemia, it is not the relevant underlying mechanism.

In an attempt to find a physiological circumstance where myocardial RIP2 may play a role, we examined contractile dysfunction in response to tumor necrosis factor and MDP. Although tumor necrosis factor induces p38-dependent contractile dysfunction (23), this was not attenuated in RIP2 null hearts (data not shown). As far as we are aware, the effects of MDP on cardiac contractility and myocardial p38 MAPK activity have not been reported previously. However, it seems likely that MDP, also a component of bacterial cell walls, contributes to septic shock syndrome independently of the lipopolysaccharide moiety (34). Because contractile depression is a prominent component of this syndrome, our findings suggest that RIP2dependent signals may contribute to the pathology and are worthy of further study.

The currently favored mechanism responsible for the SB203580-sensitive dual phosphorylation of p38 MAPK during myocardial ischemia is autoactivation $(22,31,32,36)$. This pattern of activation is thought to be promoted by the association of p38 MAPK with a scaffold protein, TGF- $\beta$-activated protein kinase 1 (TAK1) binding protein 1 (TAB1). However, this model has a number of conceptual difficulties. The first difficulty is that in $\mathrm{p} 38 \mathrm{MAPK}$, in contrast to $42 / 44$ MAPK, there is a misalignment that prevents the cooperation between $\mathrm{Lys}^{53}$ in the $\mathrm{N}$-terminal lobe and $\mathrm{Asp}^{168}$ in the C-terminal lobe, which is 
A

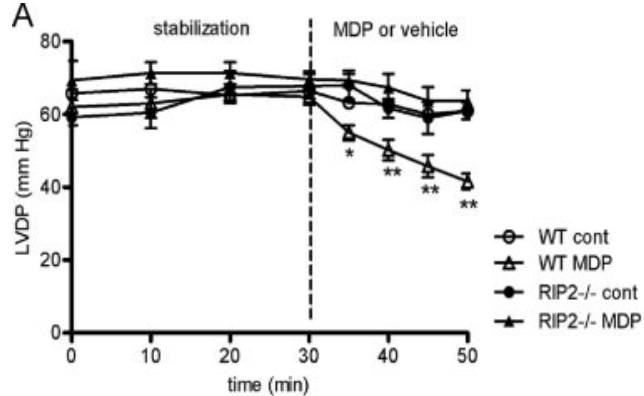

B
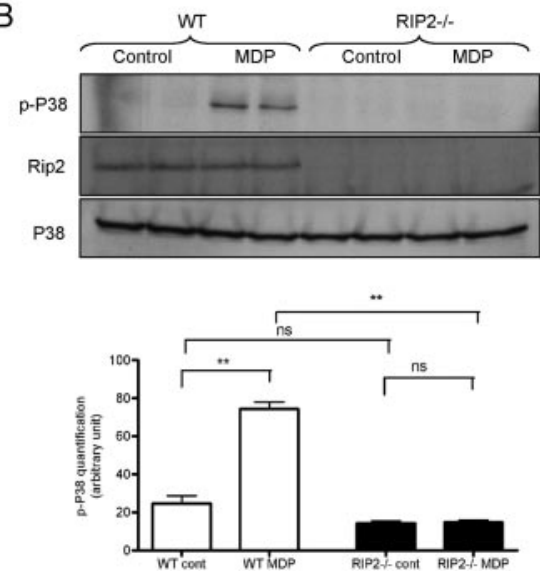
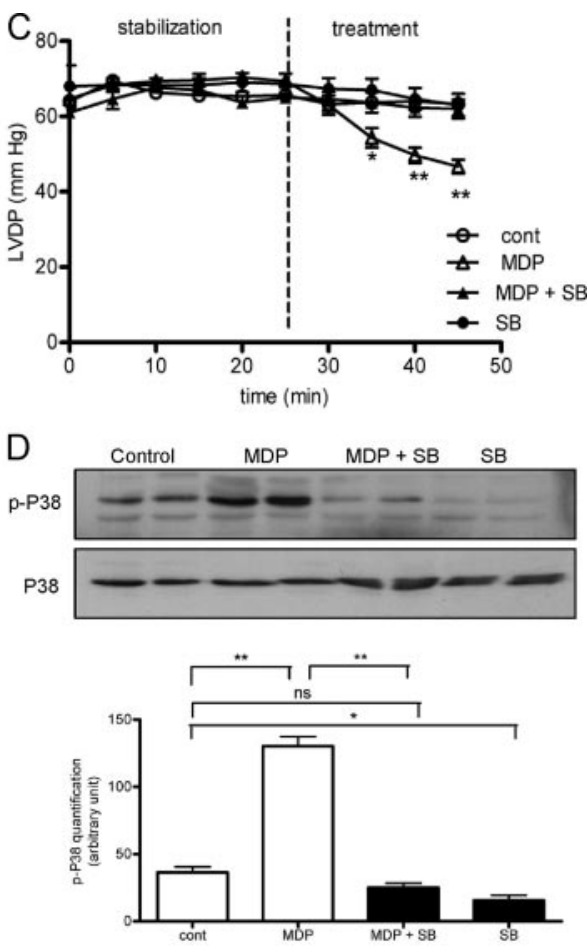

FIGURE 7. Influence of RIP2 in the myocardial response to MDP. $A$ and $B$, hearts from wild-type $(n=10)$ and RIP2-deficient (RIP2 ${ }^{-1-} ; n=6$ ) mice were retrogradely and aerobically perfused for 30 min before continued perfusion with $\mathrm{KHB}$ with or without $50 \mu \mathrm{g} / \mathrm{ml}$ muramyl dipeptide. Left ventricular contractile performance ( $L V D P$ ) was recorded throughout using a fluid-filled balloon. A depicts left ventricular developed pressure over time ( $n=3-5 /$ group). The upper panel in $B$ shows representative immunoblots prepared from MDP-exposed and control (cont)-perfused wild-type and RIP2 null hearts. The lower panel is a quantification of band density from all 16 hearts. $p$-P38, p38 MAPK. $C$ and $D$, hearts from wild-type $(n=12)$ mice were retrogradely and aerobically perfused for 30 min before continued perfusion with KHB with or without $50 \mu \mathrm{g} / \mathrm{ml}$ muramyl dipeptide. Where specified, mouse hearts were treated with $1 \mu \mathrm{M} \mathrm{SB203580}$ $(S B)$ at the end of the stabilization period. In hearts subjected to SB203580 + MDP treatment, SB203580 was added $10 \mathrm{~min}$ before and during MDP perfusion. Left ventricular contractile performance was recorded throughout using a fluid-filled balloon. $C$ depicts left ventricular developed pressure over time ( $n=3$ /group). The upper panel in $D$ shows representative immunoblots prepared from (SB203580 + MDP)-exposed and control-perfused wild-type hearts. The lower panel is a quantification of band density from all 12 hearts. ${ }^{*}, p<0.05 ;{ }^{* *}, p<0.001$. ns, not significant.

imperative to the binding and stabilization of $\operatorname{ATP}(37,38)$. Therefore, the prediction is that the non-dual phosphorylated form of p38 MAPK is inactive as a result of low ATP affinity and steric obstruction of the peptide-binding channel by the activation loop. The MKK-induced dual phosphorylation event is thought to cause the activation loop to refold (39) and move out of the peptide-binding channel. This movement is then thought to exert a "crank handle" effect on the overall tertiary structure of the kinase, reorienting the $\mathrm{N}$ - and $\mathrm{C}$-terminal lobes so that Lys $^{53}$ and $\mathrm{Asp}^{168}$ move toward one another by $2.5-5 \AA$, enabling the cooperation necessary for ATP binding (37). Thus, based on the structure-activity relationship of p38 MAPK, it is difficult to envisage how ATP binding can precede phosphorylation of the activation loop. However, such a temporal sequence is a necessary prerequisite for autoactivation. The second and perhaps more serious difficulty is that p38 MAPK is a serine/threonine kinase phylogenetically dissimilar to the dual specificity kinases possessing the ability to phosphorylate both the threonine and tyrosine residues within the activation loop. These difficulties detract from the autophosphorylation model and provide continued impetus to the identification of alternative mechanisms whereby transphosphorylation can masquerade as SB203580-sensitive autophosphorylation. Such mechanisms necessitate an SB203580-inhibitable process upstream of the dual specificity p38 MKKs. Although RIP2 fulfills many of the requirements for such a kinase, its role in p38 MAPK activation during myocardial ischemia is precluded by our findings.

The CARD protein family contains multiple members that have the ability to form heterodimers (40). Among these are proteins linked to the p38 MAPK signaling pathway, such as CARD9 (40) and TRAF6 (18). The latter, in particular, is known to activate TAK1 potentially by interacting with TAK1-binding proteins (42, 43). Thus, there are strong indications that the CARD domain of RIP2 could lead to p38 MAPK activation independent of kinase activity (41, 44). This premise is further supported by a study suggesting that the main action of the kinase domain is to stabilize RIP2 protein expression, perhaps through autophosphorylation (20). In keeping with these observations (20), we also found that the kinase-dead form of RIP2 was nearly as effective as the kinase-competent form in activating p38 MAPK. The observation of the additional action of SB203580 seen in myocytes expressing the kinase-dead form of RIP2 through the inhibition of endogenous RIP2 requires more detailed study. Thus, the SB203580sensitive downstream effects of RIP2 overexpression seen in this study, and others, are unlikely the result of simple direct pharmacological inhibition of RIP2-mediated phosphorylation of a downstream substrate.

The findings of Zhang et al. (19) suggest that RIP2 plays a role in ischemia-induced neuronal cell death. However, we found no such effect in this study, with sensitivity to infarction being similar in RIP2 null and wild-type hearts. This likely reflects the mechanisms of cell death, with necrosis and enzyme release, rather than apoptosis, dominating in myocardial infarction.

In summary, RIP2 causes SB203580-sensitive dual phosphorylation of $\mathrm{p} 38$ MAPK via TAK1 and MKKs. Although this pattern of activation resembles that seen during myocardial ischemia, we were unable to find evidence of RIP2 contributing to p38 activation or injury under this circumstance. However, RIP2 may be involved in myocardial response to MDP, and the potential role of this pathway in the contractile depression of septic shock requires further study. 


\section{REFERENCES}

1. Clark, J. E., Sarafraz, N., and Marber, M. S. (2007) Pharmacol. Ther. 116, 192-206

2. Armstrong, S. C. (2004) Cardiovasc. Res. 61, 427-436

3. Raman, M., Chen, W., and Cobb, M. H. (2007) Oncogene 26, 3100-3112

4. Tamura, K., Sudo, T., Senftleben, U., Dadak, A. M., Johnson, R., and Karin, M. (2000) Cell 102, 221-231

5. Zhang, J., Shen, B., and Lin, A. (2007) Trends Pharmacol. Sci. 28, 286-295

6. Maulik, N., Yoshida, T., Zu, Y.-L., Sat, M., Banerjee, A., and Das, D. K. (1998) Am. J. Physiol. 275, H1857-H1864.

7. Clanachan, A. S., Jaswal, J. S., Gandhi, M., Bottorff, D. A., Coughlin, J., Finegan, B. A., and Stone, J. C. (2003) Transplantation 75, 173-180

8. Yada, M., Shimamoto, A., Hampton, C. R., Chong, A. J., Takayama, H., Rothnie, C. L., Spring, D. J., Shimpo, H., Yada, I., Pohlman, T. H., and Verrier, E. D. (2004) J. Thorac. Cardiovasc. Surg. 128, $588-594$

9. Aleshin, A., Sawa, Y., Ono, M., Funatsu, T., Miyagawa, S., and Matsuda, H. (2004) Eur. J. Cardio-Thorac. Surg. 26, 974-980

10. Capano, M., and Crompton, M. (2006) Biochem. J. 395, 57-64

11. Gorog, D. A., Tanno, M., Cao, X., Bellahcene, M., Bassi, R., Kabir, A. M., Dighe, K., Quinlan, R. A., and Marber, M. S. (2004) Cardiovasc. Res. 61, 123-131

12. Wang, M., Tsai, B. M., Turrentine, M. W., Mahomed, Y., Brown, J. W., and Meldrum, D. R. (2005) Ann. Thorac. Surg. 80, 2235-2241

13. House, S. L., Branch, K., Newman, G., Doetschman, T., and Schultz, J. J. (2005) Am. J. Physiol. 289, H2167-H2175

14. Lau, J. M., Jin, X., Ren, J., Avery, J., Debosch, B. J., Treskov, I., Lupu, T. S., Kovacs, A., Weinheimer, C., and Muslin, A. J. (2007) Mol. Cell. Biol. 27, 1455-1466

15. Kang, Y. J., Seit-Nebi, A., Davis, R. J., and Han, J. (2006) J. Biol. Chem. 281, 26225-26234

16. Godl, K., Wissing, J., Kurtenbach, A., Habenberger, P., Blencke, S., Gutbrod, H., Salassidis, K., Stein-Gerlach, M., Missio, A., Cotten, M., and Daub, H. (2003) Proc. Natl. Acad. Sci. U. S. A. 100, 15434-15439

17. Argast, G. M., Fausto, N., and Campbell, J. S. (2005) Mol. Cell Biochem. 268, $129-140$

18. McCarthy, J. V., Ni, J., and Dixit, V. M. (1998) J. Biol. Chem. 273, $16968-16975$

19. Zhang, W. H., Wang, X., Narayanan, M., Zhang, Y., Huo, C., Reed, J. C., and Friedlander, R. M. (2003) Proc. Natl. Acad. Sci. U.S.A. 100, $16012-16017$

20. Windheim, M., Lang, C., Peggie, M., Plater, L. A., and Cohen, P. (2007) Biochem. J. 404, 179-190

21. Kobayashi, K., Inohara, N., Hernandez, L. D., Galan, J. E., Nunez, G., Janeway, C. A., Medzhitov, R., and Flavell, R. A. (2002) Nature 416, 194-199

22. Tanno, M., Bassi, R., Gorog, D. A., Saurin, A. T., Jiang, J., Heads, R. J., Martin, J. L., Davis, R. J., Flavell, R. A., and Marber, M. S. (2003) Circ. Res. 93, 254-261

23. Bellahcene, M., Jacquet, S., Cao, X. B., Tanno, M., Haworth, R. S., Layland, J., Kabir, A. M., Gaestel, M., Davis, R. J., Flavell, R. A., Shah, A. M., Avkiran,
M., and Marber, M. S. (2006) J. Am. Coll. Cardiol. 48, 545-555

24. Eickhoff, J., Hanke, M., Stein-Gerlach, M., Kiang, T. P., Herzberger, K., Habenberger, P., Muller, S., Klebl, B., Marschall, M., Stamminger, T., and Cotten, M. (2004) J. Biol. Chem. 279, 9642-9652

25. Punn, A., Mockridge, J. W., Farooqui, S., Marber, M. S., and Heads, R. J. (2000) Biochem. J. 350, 891-899

26. Ge, B., Gram, H., Di Padova, F., Huang, B., New, L., Ulevitch, R. J., Luo, Y., and Han, J. (2002) Science 295, 1291-1294

27. Lu, C., Wang, A., Dorsch, M., Tian, J., Nagashima, K., Coyle, A. J., Jaffee, B., Ocain, T. D., and Xu, Y. (2005) J. Biol. Chem. 280, 16278-16283

28. Dorsch, M., Wang, A., Cheng, H., Lu, C., Bielecki, A., Charron, K., Clauser, K., Ren, H., Polakiewicz, R. D., Parsons, T., Li, P., Ocain, T., and Xu, Y. (2006) Cell. Signal. 18, 2223-2229

29. Ninomiya-Tsuji, J., Kajino, T., Ono, K., Ohtomo, T., Matsumoto, M., Shiina, M., Mihara, M., Tsuchiya, M., and Matsumoto, K. (2003) J. Biol. Chem. 278, 18485-18490

30. Marber, M. S. (2000) Circ. Res. 86, 926-931

31. Li, J., Miller, E. J., Ninomiya-Tsuji, J., Russell, R. R., III, and Young, L. H. (2005) Circ. Res. 97, 872-879

32. Fiedler, B., Feil, R., Hofmann, F., Willenbockel, C., Drexler, H., Smolenski, A., Lohmann, S. M., and Wollert, K. C. (2006) J. Biol. Chem. 281, 32831-32840

33. Iwanaga, Y., Davey, M. P., Martin, T. M., Planck, S. R., DePriest, M. L., Baugh, M. M., Suing, C. M., and Rosenbaum, J. T. (2003) Inflamm. Res. 52, 272-276

34. Murch, O., Abdelrahman, M., Kapoor, A., and Thiemermann, C. (2008) Shock, in press

35. Vahebi, S., Ota, A., Li, M., Warren, C. M., de Tombe, P. P., Wang, Y., and Solaro, R. J. (2007) Circ. Res. 100, $408-415$

36. Ge, B., Xiong, X., Jing, Q., Mosley, J. L., Filose, A., Bian, D., Huang, S., and Han, J. (2003) J. Biol. Chem. 278, 2286-2293

37. Wilson, K. P., Fitzgibbon, M. J., Caron, P. N., Griffith, J. P., Chen, W. Y., McCaffrey, P. G., Chambers, S. P., and Su, M. S.-S. (1996) J. Biol. Chem. 271, 27696-27700

38. Gum, R. J., McLaughlin, M. M., Kumar, S., Wang, Z. L., Bower, M. J., Lee, J. C., Adams, J. L., Livi, G. P., Goldsmith, E. J., and Young, P. R. (1998) J. Biol. Chem. 273, 15605-15610

39. Canagarajah, B. J., Khokhlatchev, A., Cobb, M. H., and Goldsmith, E. J. (1997) Cell 90, 859-869

40. Hsu, Y. M., Zhang, Y., You, Y., Wang, D., Li, H., Duramad, O., Qin, X. F., Dong, C., and Lin, X. (2007) Nat. Immunol. 8, 198-205

41. Yang, Y., Yin, C., Pandey, A., Abbott, D., Sassetti, C., and Kelliher, M. A. (2007) J. Biol. Chem. 282, 36223-36229

42. Kanayama, A., Seth, R. B., Sun, L., Ea, C. K., Hong, M., Shaito, A., Chiu, Y. H., Deng, L., and Chen, Z. J. (2004) Mol. Cell 15, 535-548

43. Wang, W. P., Chen, J. X., Liao, R., Deng, Q. D., Zhou, J. J., Huang, S., and Sun, P. Q. (2002) Mol. Cell. Biol. 22, 3389-3403

44. Hasegawa, M., Fujimoto, Y., Lucas, P. C., Nakano, H., Fukase, K., Nunez, G., and Inohara, N. (2007) EMBO J. 27, 373-383 